\title{
DETERMINAÇÃO DA CONCENTRAÇÃO DE $\beta$-GLUCANO EM COGUMELO Agaricus blazei Murill POR MÉTODO ENZIMÁTICO'
}

\author{
Yong K. PARK ${ }^{2, *}$, Masaharu IKEGAKI ${ }^{2}$, Severino M. ALENCAR ${ }^{2}$, Claudio L. AGUIAR ${ }^{2}$
}

\begin{abstract}
RESUMO
Cogumelos comestiveis contêm importantes propriedades funcionais. Em particular, $\beta$-glucanos, homo- e hetero-glucanos com ligações glicosídicas $\beta(1 \rightarrow 3), \beta(1 \rightarrow 4)$ e $\beta(1 \rightarrow 6)$, supostamente responsáveis por algumas propriedades benéficas à saúde humana, como atividade imunomodulatória, antioxidante, antiinflamatória e anticancerígena. Neste trabalho, a quantidade de $\beta$-glucano presente em cogumelo Agaricus blazei Murill, coletados de três diferentes locais, foi analisada utilizando-se método enzimático. As amostras (em base seca) foram tratadas com $\alpha$-amilase, protease bacteriana e com glicoamilase fúngica. $\beta$-glucano foi quantificado após hidrólise ácida e determinação da glicose liberada. Foi verificado que amostras de A. blazei Murill cultivadas em estufas apresentaram menor concentração de $\beta$-glucano $(8,4 \pm 0,9$ e $7,6 \pm 2,8 \mathrm{~g} / 100 \mathrm{~g})$ quando comparado com amostras cultivadas em campo aberto (10,1 $\pm 2,1 \mathrm{~g} / 100 \mathrm{~g})$. Observou-se ainda que, mesmo sendo cultivado em condições semelhantes, porém em locais diferentes, as amostras apresentaram diferenças significativas $(7,6 \pm 2,8$ e $8,4 \pm 0,9 \mathrm{~g} / 100 \mathrm{~g})$.

Palavras-chave: $\beta$-glucano; determinação enzimática; Agaricus blazei Murill.
\end{abstract}

\section{SUMMARY}

DETERMINATION OF $\beta$-GLUCAN CONCENTRATION IN Agaricus blazei Murill MUSHROOM BY ENZYMATIC METHOD. Edible mushrooms contains a very interesting functional properties. Among them, the $\beta$-glucans, polysaccharides with $\beta-1,3 ; \beta-1,4$ and $\beta-1,6$ glucosidic linkages, they are responsible to a series of properties to human health, such as immunomodulatory, antioxidant, antiinflammatory and, antitumoral activities. In the present work, the Agaricus blazei Murill $\beta$-glucan concentrations from three locations, were determined through the enzymatic method. Samples were treated with $\alpha$-amylase, bacterial protease and fungal glucoamylase. $\beta$-glucans were quantified after acid hydrolysis and, the glucose determined for spectrophotometric method. It was verified that samples cultivated inside stoves presented smaller $\beta$-glucan concentration $(8.4 \pm 0.9$ and $7.6 \pm 2.8 \mathrm{~g} / 100 \mathrm{~g})$, when compared with samples cultivated in open field $(10.1 \pm 2.1 \mathrm{~g} / 100 \mathrm{~g})$. It was also observed that the samples cultivated in similar conditions, even so in different place, presented significant differences $(7.6 \pm 2.8$ and $8.4 \pm 0.9 \mathrm{~g} / 100 \mathrm{~g})$.

Keywords: $\beta$-glucan; enzymatic determination; Agaricus blazei Murill.

\section{1 - INTRODUÇÃO}

A maioria dos cereais contém quantidades variáveis de $\beta-(1 \rightarrow 3)$ e $\beta-(1 \rightarrow 4)-D$-glucanos. Esses polissacarideos são comuns entre os cereais e ocorrem em grande quantidade no endosperma e na parede celular de cevada, trigo e aveia $[2,11]$. A importância metabólica do $\beta$-glucano na saúde e na nutrição humana foi recentemente revisada por KLOPFENSTEIN [15]. Alimentos à base de aveia, ricos em fibras solúveis em água têm sido descritos como produtos hipocolesterotêmicos em humanos e, tem despertado interesse crescente junto à comunidade médica [1, 27]. O $\beta$-glucano (Figura 1) é encontrado em todos cereais, mas as concentrações são maiores em aveia e cevada, com valores na faixa de 2 a $6 \%$ [10]. Os cogumelos comestiveis também apresentam compostos que têm propriedades funcionais, em particular os homo e hetero-glucanos com ligações glicosídicas do tipo $\beta(1 \rightarrow 3)$ e $\beta(1 \rightarrow 6)$ [16]. No entanto, além das propriedades farmacológicas encontradas nos polissacarídeos de origem vegetal, os polissacarídeos de origem fúngica apresentam várias outras propriedades tais como atividades antitumoral, imunomodulatória, antiviral, antimicrobiana e antiparasitária [3, 9, 34]. Entre

${ }^{1}$ Recebido para publicação em 21/12/2000. Aceito para publicação em 23/04/2003 (000557).

${ }^{2}$ Universidade Estadual de Campinas, Faculdade de Engenharia de Alimentos, Laboratório de Bioquímica, Caixa Postal, 6177, CEP. 13083970, Campinas/SP, Brasil.E-mail:ykpark@fea.unicamp.br

* A quem a correspondência deve ser enviada. as atividades farmacológicas relacionadas a diversas espécies de cogumelos que contém $(1 \rightarrow 3)$ e $(1 \rightarrow 6)-\beta-D$ glucano, está a atividade antitumoral $[3,9,14,16,19,20$, 21]. De acordo com KAWAGISH et al. [13, 14] a fração de polissacarídeo estraído de Agaricus blazei, que apresenta atividade antitumoral é composta de um complexo de $\beta-(1 \rightarrow 6)-D$-glucano e proteínas.

O objetivo deste trabalho foi avaliar a concentração de $\beta$-glucano em cogumelos Agaricus blazei Murill cultivados no Brasil e no Japão em duas condições distintas (estufa e campo) através de método descrito por PROSKY et al. [29] e modificado pela "Foundation of Japanese Food Analysis Center" [8], pela quantificação de glicose liberada após sucessivas hidrólises enzimática e química.

\section{2 - MATERIAL E MÉTODOS}

\section{1 - Amostras}

As amostras de cogumelos foram cultivadas no Brasil e no Japão, e em duas condições distintas de acordo com a Tabela 1. O cogumelo foi cultivado em estufa a $25 \pm 0,2^{\circ} \mathrm{C}$ e umidade relativa (U.R.) superior a 90\%, por 6 dias, sobre composto orgânico produzido a partir de bagaço de can $\alpha$-deaçúcar, farelo de arroz, esterco de gado e galinha, contidos em sacos plásticos de 60 quilos. Para a amostra cultivada no campo, a incubação foi feita em composto orgânico coberto com terra estéril, em valas, a temperatura ambiente, a temperatura de $25 \pm 2^{\circ} \mathrm{C}$ e U.R. aproximada de $60 \%$. 
(A1)

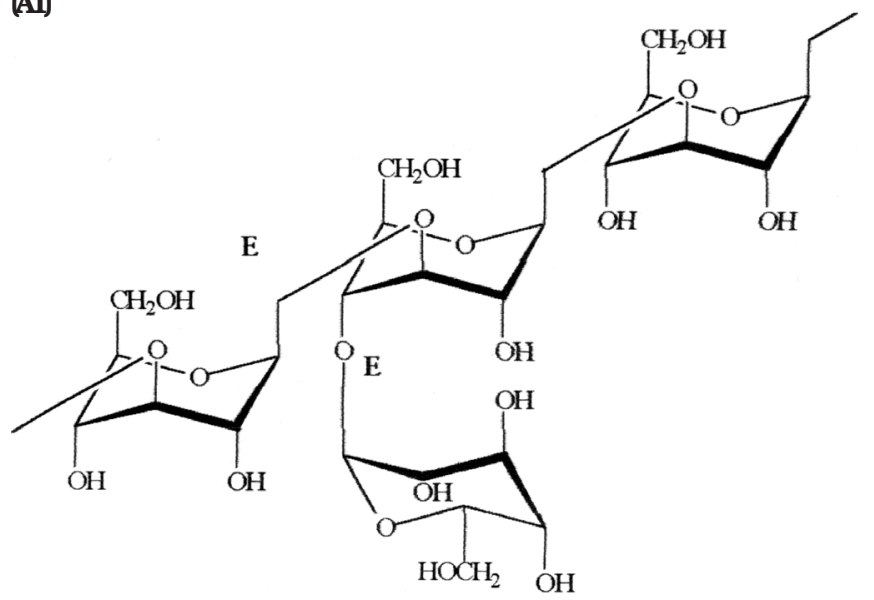

(A2)

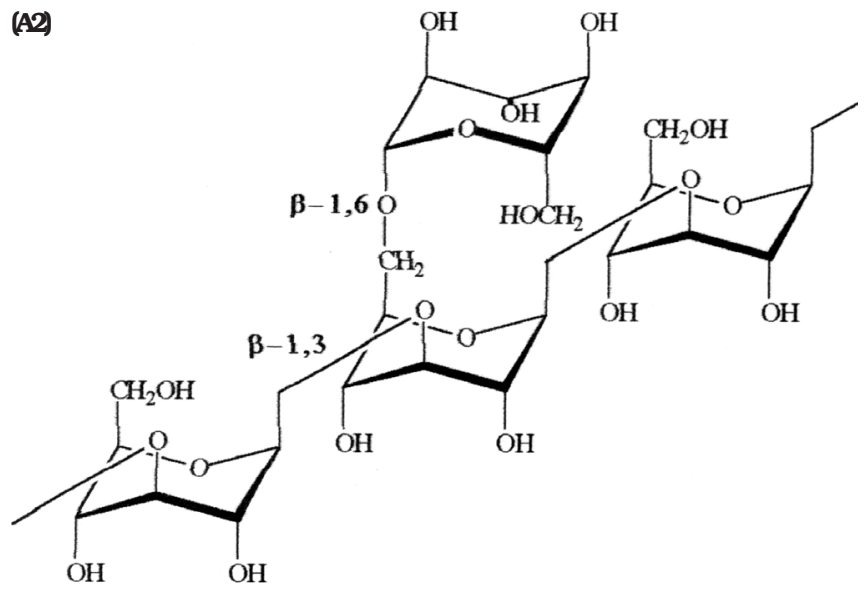

(B)

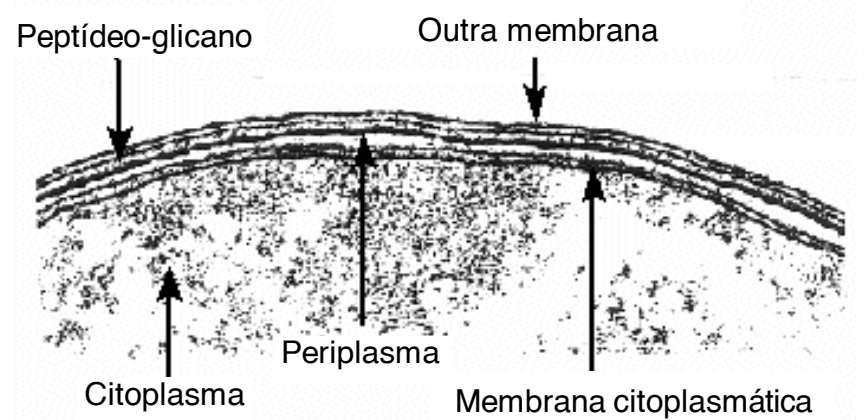

FIGURA 1. (A1 e A2) Unidades estruturais de $\beta$-glucanos encontrados em cereais e microrganismos e (B) estrutura da parede celular de leveduras.

TABELA 1. Locais e condições de cultivo do cogumelo Agaricus blazei Murill

\begin{tabular}{ccccc}
\hline Amostras & Local & Condição & Umidade relativa $(\%)$ & Temperatura $\left({ }^{\circ} \mathrm{C}\right)$ \\
\hline$J$ & Japão & Estufa & $>90$ & $25 \pm 0,2$ \\
$C$ & São Paulo & Estufa & $>90$ & $25 \pm 0,2$ \\
$R$ & São Paulo & Campo & $50-60$ & $25 \pm 2,0$ \\
\hline
\end{tabular}

\section{2 - Preparo do cogumelo}

As amostras foram coletadas, lavadas e imediatamente colocadas em estufa com circulação forçada a $60^{\circ} \mathrm{C}$ por $24 \mathrm{~h}$, para a secagem do cogumelo. Terminado o processo de desidratação, as amostras foram acondicionadas em dessecador e armazenadas em freezer ($10^{\circ} \mathrm{C}$ ) até o momento de sua análise. As amostras foram pulverizadas utilizando um triturador LI-5 (capacidade de 5L e motor de 3/4 Hp, Reimse Co.), peneiradas $(0,3 \mathrm{~mm})$ e imediatamente analisadas.

\section{3 - Determinação do $\beta$-glucano}

A determinação de $\beta$-glucano foi realizada em triplicata, conforme a metodologia descrita por PROSKY et al. [29] e modificada pela "Foundation of Japanese Food Analysis Center" [8]. Esse método consiste em hidrólises enzimáticas e químicas, e constitui o método analítico oficial japonês para determinação de $\beta$ glucano. Aliquotas de $1 \mathrm{~g}$ das amostras desidratadas e trituradas, foram acondicionadas em frascos de Erlenmeyer (500mL) com $50 \mathrm{~mL}$ de tampão fosfato $80 \mathrm{mM}$ e pH 6,0. Em seguida, foram tratadas com: (a) $\alpha$-amilase termostável Termamyl 120L (Novo Nordisk; 0,1 mL de enzima, incubada por 30min em banho-maria em ebulição), (b) protease neutra bacteriana (Novo Nordisk; $0,1 \mathrm{~mL}$ de enzima, pH 7,5; ajustado com $\mathrm{NaOH} 25 \mathrm{mM}$, incubado por $30 \mathrm{~min}$ a $60^{\circ} \mathrm{C}$ ) e (c) amiloglicosidase AMG 200L (Novo Nordisk; 0,3mL de enzima, pH 4,0-4,5, ajustado com $\mathrm{HCl} 75 \mathrm{mM}$, incubado por $30 \mathrm{~min}$ a $60^{\circ} \mathrm{C}$ ). Após os tratamentos enzimáticos, foram adicionados $200 \mathrm{~mL}$ de solução $95 \%$ de álcool etílico, e os frascos foram mantidos a $60^{\circ} \mathrm{C}$ por 60 minutos. Em seguida, as fibras solúveis precipitadas com etanol e fibras insolúveis foram separadas por centrifugação a $20000 \mathrm{~g}$ por 10 minutos. Os precipitados lavados com solução $80 \%$ de álcool etilico e acetona, continham sais insolúveis, proteínas não-digeridas e fibras insolúveis, como descrito por MANZI \& PIZZOFERRATO [16]. O procedimento de lavagem dos precipitados foi repetido por 3 vezes. Após evaporação do etanol e da acetona excedentes foram adicionados $10 \mathrm{~mL}$ de solução $72 \%$ de $\mathrm{H}_{2} \mathrm{SO}_{4}$ e os frascos foram incubados a $20^{\circ} \mathrm{C}$ por $4 \mathrm{~h}$, para hidrólise das fibras, compostas basicamente de $\beta$-glucanos. Em seguida, foram adicionados $140 \mathrm{~mL}$ de água destilada, sendo os frascos incubados em banho-maria em ebulição por $2 \mathrm{~h}$. Após este periodo, o pH das misturas foi ajustado até a neutralidade utilizando $\mathrm{NaOH} 5 \mathrm{M}$, e o volume final completado para $300 \mathrm{~mL}$. Em seguida as amostras foram filtradas em papel de filtro Whatman $\mathrm{n}-5 \mathrm{~B}$ Advantec e a quantidade de glicose foi determinada pelo método enzimático utilizando-se 4-aminofenazona $(0,025 \mathrm{~mol} / \mathrm{L})$ e fenol $(0,055 \mathrm{~mol} / \mathrm{L})$ na presença de glicose oxidase $(1 \mathrm{U} / \mathrm{mL})$ e peroxidase $(0,15 \mathrm{U} / \mathrm{mL})$ (LaborLab Ltda., Guarulhos/SP). A concentração de $\beta$ glucano (g/100g), presente nos cogumelos em base seca, foi calculada através da equação:

$\beta$-glucano $(\mathrm{g} / 100 \mathrm{~g})=$ Glicose $(\mathrm{g} / 100 \mathrm{~g}) \times 0,9$ 


\section{4 - Análise estatística}

O delineamento empregado foi inteiramente casualizado, sendo que a análise estatística dos resultados obtidos de 3 repetições. Os dados obtidos da concentração de $\beta$-glucano foram analisados pelo programa MSTAT-C [22] para a determinação da análise da variância e comparação de médias através do teste de Tukey com um nivel de confiança de 95\%.

\section{3 - RESULTADOS E DISCUSSÃO}

Utilizando-se a metodologia da determinação de $\beta$-Glucano proposta por PROSKY et al. [29] e modificada pela "Foundation of Japanese Food Analysis Center" [8], as amostras de cogumelos foram submetidas a tratamento com $\alpha$-amilase bacteriana Termamyl, para a hidrólise do amido seguidas com protease e com amiloglicosidase para hidrólise de olissácarídeos residuais para remoção de proteínas digeriveis. O resíduo obtido foi lavado para a remoção de sais insolúveis, proteinas não digeridas e fibras insolúveis. As amostras de $\beta$ glucano foram hidrolisadas com ácido e a quantidade de glicose formada foi determinada pelo método enzimático com glicose oxidase e peroxidase. O uso de enzimas altamente purificadas é requerido na análise de $\beta$-glucano em alimentos, pois a contaminação das preparações enzimáticas com enzimas que hidrolisam o amido, pode resultar na produção de açúcares livres, como glicose de outra fonte que não seja do $\beta$-glucano, e isto poderia superestimar o conteúdo de $\beta$-glucano. A glicose oxidase e peroxidase usadas na determinação da glicose devem ser essencialmente livres de catalase e $\alpha$-e $\beta$-glicosidase [18]. Por este motivo, as preparações enzimáticas utilizadas neste trabalho foram obtidas de fornecedores que garantissem a pureza requerida. Além disto, a metodologia utilizada foi inicialmente elaborada para determinação do conteúdo de $\beta$-glucano em cereais, onde o teor das fontes potenciais de glicose, como amido ou celulose/hemicelulose, são consideravelmente inúmeras. O cogumelo Agaricus blazei, possui teor reduzido, senão inexistente destes polissacarideos; e neste trabalho foram utilizadas enzimas puras no tratamento enzimático das amostras trituradas de A. blazei. Como sugerido por McCLEARY [17] o método proposto por McCLEARY para análise de $\beta$ glucanos, tem sido aplicado pelo Sub-Comitê do "Cereal Chemistry Division of the Royal Australian Chemical Institute", e atualmente é recomendado como método australiano [33]. DALIES et al. [6] afirmam que a parede celular de fungos e leveduras, são compostas principalmente, de $\beta$-glucanos, que hidrolisados levariam à formação de glicose, e de mananas e quitina (Figura 2), que hidrolisados levariam a formação de outras hexoses, como manose, e N-acetilglicosamina. A composição da parede celular de Fusarium oxysporum foi analisada por SCHOFFELMEER et al. [32], sendo encontrados principalmente, quitina, $\alpha-$ e $\beta$-glucanos, e a análise de açúcares destes polissacarídeos mostrou não somente a presença de glicose e $\mathrm{N}$-acetilglicosamina, mas também de manose, galactose e ácido urônico. Algumas algas e oomicetos possuem ainda celulose em suas paredes celulares, que poderiam, após tratamento enzimático, formar um excedente de glicose que acarretaria em valores superestimados de $\beta$-glucanos.

Em geral, os métodos descritos na literatura para determinação de $\beta$-glucanos, foram desenvolvidos para análise de $\beta$-glucanos de cereais com ligações do tipo $\beta(1 \rightarrow 3)(1 \rightarrow 6)[18]$. De fato, cereais contêm essencialmente $\beta$-glucanos com ligações mistas de $\beta(1 \rightarrow 3)(1 \rightarrow 6)$, enquanto cogumelos apresentam maiores quantidades de $\beta(1 \rightarrow 4)(1 \rightarrow 6)$ que $\beta(1 \rightarrow 3)(1 \rightarrow 4)$ e diferentes ligações simples como $\beta(1 \rightarrow 3), \beta(1 \rightarrow 4)$ e $\beta(1 \rightarrow 6)$ [23]. Na Tabela 2 , são apresentados os teores de $\beta$-glucano presentes nas amostras de cogumelo A. blazei em duas condições de cultivo. Os teores de $\beta$-glucano representam os valores médios de três avaliações e a comparação entre as médias foi feita considerando-se 5\% de significância.

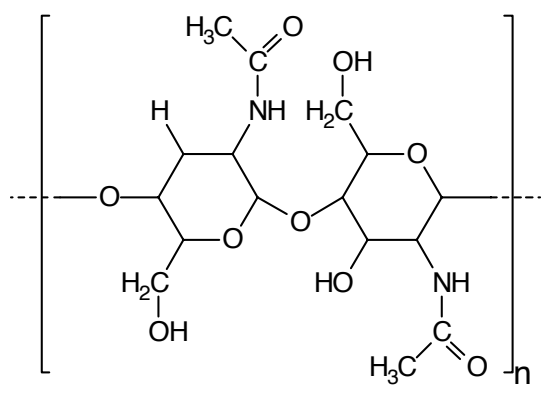

FIGURA 2. Estrutura química de quitina encontrados em microrganismos.

TABELA 2. Concentração de $\beta$-glucano das amostras de cogumelo Agaricus blazei Murill cultivadas no Brasil e no Japão em diferentes condições*

\begin{tabular}{ccc}
\hline Amostra & Local/Condição & $\beta$-glucano** \\
\hline$J$ & Japão (estufa) & $7,6 \pm 2,8^{\mathrm{C}}$ \\
$C$ & São Paulo (estufa) & $8,4 \pm 0,9^{\mathrm{B}}$ \\
$R$ & São Paulo (campo) & $10,1 \pm 2,1^{\mathrm{A}}$ \\
\hline
\end{tabular}

${ }^{*}$ Médias seguidas de letras diferentes são significativas em nivel de $5 \%$ (Teste de Médias seguidas de letras diferentes são significativas em nivel de $5 \%$ (Teste de
Tukey; p<0,05) de $\beta$-glucano por $100 \mathrm{~g}$ de cogumelo, em base seca.

As amostras cultivadas em estufa, tanto no Brasil como no Japão, apresentaram menor concentração de $\beta$-glucano quando comparadas com a amostra cultivada em campo e, observou-se ainda que, houve diferença significativa no teor de $\beta$-glucano entre as duas amostras cultivadas em estufa.

Esta diferença pode ter sido causada devido à variação na qualidade do composto utilizado para o cultivo do cogumelo bem como alterações nas condições ambientais dentro das estufas. Verificou-se ainda que comparando as amostras $\mathrm{C}$ e $\mathrm{R}$, cultivadas em estufa e no campo, respectivamente, houve diferença significativa no teor de $\beta$-glucano entre as duas formas de cultivo, levando a concluir que a melhor concentração deste polissacarídeo 
pode ser obtida se o cogumelo for cultivado no campo. Nesse caso existem alguns fatores como chuva, variação brusca de temperatura, pragas, etc, que podem afetar de forma negativa o cultivo dos cogumelos. Estes fatores agrícolas, têm afetado o teor de $\beta$-glucano, quando se trata de cultivo de cereais, porém o efeito destes fatores agricolas nã foram analisados para o cultivo de cogumelos em campo. Algum estudo neste sentido poderia ser feito, para que se possa elaborar um protocolo de produção destes cogumelos com um teor de $\beta$-glucano que seja maior e invariável.

MANZI \& PIZZOFERRATO [16] utilizaram método analitico similar ao empregado neste trabalho e encontraram teores de $\beta$-glucano em cogumelos comestiveis Pleurotus ostreatus, P. pulmunarius, P. eryngii e Lentinula edodes na faixa de 0,21 e 0,53g/100g de cogumelo seco. Outros autores, como DALLIES et al. [6] por sua vez, avaliaram teores de $\beta$-glucanos em Saccharomyces cerevisiae, enquanto SANDULA et al. [31] avaliaram a presença de $\beta(1 \rightarrow 3)$-glucano nas paredes celulares de Saccharomyces cerevisiae e de Aspergillus niger. NGUYEN et al. [24] afirmaram que a parede celular de leveduras como Kluyveromyces marxianus, Kloeckera apiculata, Debaryomyces hansenii, Zygosaccharomyces bailii e Saccharomyces cerevisiae, representa de 26-32\% da massa seca do organismo, e que as manoproteinas representam $25-34 \%$ da parede celular, os glucanos insolúveis em álcali, de 15-48\%, e por último, os glucanos solúveis em álcali, de 10-48\%. CHEUNG \& LEE [4] analisaram Pleurotus tuber e encontraram concentrações de fibras totais solúveis em álcali de 126 e 293g/kg de cogumelo, sendo que destas fibras foram encontrados principalmente $\beta$-glucano (ligações do tipo $\beta-1,3, \beta-1,4$ e $\beta-1,6$ ) e quitina. No método proposto por OHNO et al. [25] para extração de $\beta$ - $(1 \rightarrow 3)$-glucano solúvel de Candida spp. pela oxidação com hipoclorito de sódio e subseqüente extração com dimetilsulfóxido, foi obtido $9,6 \%$ de $\beta$-glucano, que continha principalmente ligações do tipo $\beta-1,3$ e $\beta-1,6$.

Em termos comparativos, em grãos, diferentes trabalhos apresentam teores variáveis para o teor de $\beta$ glucano em grãos de cereais, como aveia e cevada. SAASTAMOINEN et al. [30] encontraram teores médios de $\beta$-glucano em aveia variando de 1,9 a $5,1 \%$ (p/v). CHO $\&$ WHITE [5] relataram que o conteúdo de $\beta$-glucano em grãos de 243 amostras de aveia, mostraram uma distribuição normal com a maioria das amostras em torno de 4,5-5,5\% de $\beta$-glucano por $100 \mathrm{~g}$ de aveia seca. Os autores verificaram que a variabilidade genética dos cultivares afetou o conteúdo de $\beta$-glucano. O mesmo foi observado por PETERSON et al. [28] e OUARNIER et al. [26], que também verificaram que o conteúdo de $\beta$-glucano em aveia (Avena sativa) variou com a época de colheita entre outros fatores agricolas. Um conteúdo maior foi encontrado por WELCH et al. [35], quando analisaram Avena atlantica, a qual apresentou teores de 2,2 a 11,3g de $\beta$-glucano por $100 \mathrm{~g}$ do grão. Em grãos de cevada, estes valores podem chegar a 3,5-4,8\% [7]. Estas variações segundo HUBIK \& TICHY [12], estão associadas ao genótipo e local em que o cereal foi cultivado. Fatores como a fertilização do solo não mostraram ter efeito significativo no aumento de $\beta$-glucano em cereais.

\section{4 - CONCLUSÕES}

A concentração de $\beta$-glucano em cogumelo Agaricus blaze variou significativamente conforme a forma de cultivo, sendo que os cogumelos cultivados em São Paulo, em estufa apresentavam menor concentração de $\beta$-glucano $(8,4 \pm 0,9 \mathrm{~g} / 100 \mathrm{~g}$ de cogumelo em base seca), quando comparado com amostras cultivadas no campo $(10,1 \pm 21 \mathrm{~g} / 100 \mathrm{~g}$ de cogumelo em base seca).

\section{5 - REFERÊNCIAS BIBLIOGRÁFICAS}

[1] ANDERSON, J. W.; STORY, L.; SIELING, B.; CHEN, W. J.L.; PETRO, M. S.; STORY, J. Hypocholesterolemic effects of oat-bran or bean intake for hypercholesterolemic meno. American Journal Clinical Nutrition, v. 40, n. 6, p. 1146-1155, 1984.

[2] CARR, J. M.; GLATTER, S.; JERACI, J. L.; LEWIS, B. A. Enzymatic determination of $\beta$-glucan in cereal-based food-products. Cereal Chemistry, v. 67, n. 3, p. 226229, 1990.

[3] CHEUNG, P. C.K. Plasma and hepatic cholesterol levels and fecal neutral sterol excretion are altered in hamsters fed straw mushroom diets. Journal of Nutrition, v. 128, n. 9, p. 1512-1516, 1998.

[4] CHEUNG, P. C.K.; LEE, M.Y. Fractionation and characterization of mushroom dietary fiber (nonstarch polysaccharides) as potential nutraceuticals from sclerotia of Pleurotus tuber-regium (Fries) Singer. Journal of Agricultural and Food Chemistry, v. 48, n. 8, p. 3148-3151, 2000.

[5] CHO, K.C.; WHITE, P. J. Enzymatic analysis of bet $\alpha$-glucan content in different oat genotypes. Cereal Chemistry, v. 70, n. 5, p. 539-542, 1993.

[6] DALLIES, N. ; FRANCOIS, J.; PAQUET, V. A new method for quantitative determination of polysaccharides in the yeast cell wall: Application to the cell wall defective mutants of Saccharomyces cerevisiae. Yeast, v. 14, n. 14, p. 1297-1306, 1998.

[7] ENGSTROM, D.F.; MATHISON, G.W.; GOONEWARDENE, L.A. Effect of bet $\alpha-$ glucan, starch, and fiber content an steam vs dry rolling of barley-grain on its degradability and utilization by steers. Animal Feed Science and Technology, v. 37, n. 1-2, p. 33-46, 1992.

[8] FOUNDATION OF JAPANESE FOOD ANALYSIS CENTER, No 398040072-001 52-1, Yoyogi-Machi, Shibuy $\alpha-\mathrm{Ku}$, Tokyo, Japan. 1980.

[9] FUJIMIYA, Y.; KOBORI, H.; OSHIMAN, K.; SODA, R.; EBINA, T. Tumoricidal activity of high molecular weight polysaccharides derived from Agaricus blazei via oral administration in the mouse tumor model. Journal of Japan Society of Food Science, v. 45, n. 4, p. 246-252, 1998.

[10] GENÇ, H.; OZDEMIR, M.; DEMIRBAS, A. Analysis of mixed-linked (1-3), (1-4)-B-D-glucans in cereals grains from Turkey. Food Chemistry, v. 73, n. 3, p. 221-224, 2001.

[11] HENRY, R. J. Pentosan and (1-3),(1-4)-bet $\alpha$-glucan concentrations in endosperm and whole grain of wheat, barley, oats and rye. Journal of Cereal Science, v. 6, n. 3, p. 253-258, 1987.

[12] HUBIK, K.; TICHY, F. Effects of ecological and cropping factors on bet $\alpha$-glucan content in oats. Rostlinna Vyroba, v. 42, n. 1, p. 29-33, 1996. 
[13] KAWAGISHI, H.; KATSUMI, R.; SAZAWA, T.; MIZUNO, T.; HAGIWARA, T.; NAKAMURA, T. Cyto-toxic steroids from the mushroom Agaricus blazei. Phytochemistry, v. 27, n. 9, p. 2777-2779, 1988.

[14] KAWAGISHI, H.; NOMURA, A.; YUMEN, T.; MIZUNO, T.; HAGIWARA, T.; NAKAMURA, T. Isolation and properties of a lectin from the fruiting bodies of Agaricus blazei. Carbohydrate Research, v. 186, n. 2, p. 267-273, 1989.

[15] KLOPFENSTEIN, C. F. The role of cereal $\beta$-glucans in nutrition and health. Cereal Foods World, v. 33, n. 10, p. 865, 1988.

[16] MANZI, P. ; PIZZOFERRATO, L. $\beta$-glucans in edible mushrooms. Food Chemistry, v. 68, n. 3, p. 315-318, 2000.

[17] McCLEARY, B. V. Importance of enzyme purity and activity in the measurement of total dietary fiber and dietary fiber components. Journal of Association of Official Analytical Chemistry International, v. 83, n. 4, p. 9971005, 2000.

[18] McCLEARY, B. V.; HOLMES, M. G. Enzymic quantification of $(1-3),(1,4) \beta$-glucan in barley and malt. Journal of the Institute of Brewing, v. 91, n. 5, p. 285-295, 1985.

[19] MIZUNO, T. A development of antitumor polysaccharides from mushroom fungi. Food and Food Ingredient Japanese Journal, v. 167, p. 69-85, 1996.

[20] MIZUNO, T.; HAGIWARA, T.; NAKAMURA, T.; ITO, H.; SHIMURA, K.; SUMIYA, T.; ASAKURA, A. Studies on the host-mediated antitumor polysaccharides. 13. Antitumor activity and some properties of waterinsoluble hetero-glycans from "Himematsutake", the fruiting body of Agaricus blazei Murill. Agricultural and Biological Chemistry, v. 54 , n. 11 , p. 28892896, 1990.

[21] MIZUNO, T.; MORIMOTO, M.; MINATO, K.; TSUCHIDA, H. Polysaccharides from Agaricus blazei stimulate lymphocyte T-cell subsets in mice. Bioscience, Biotechnology and Biochemistry, v. 62, n. 3, p. 434437, 1998.

[22] MSTAT-C. A microcomputer program for the design, management and analysis of agronomic research experiments. Norway: MSTAT Distribution, n. p. , 1998.

[23] MULlins, J. T. Regulatory mechanism of $\beta$-glucan synthetases in bacteria, fungi and plants. Physiological Plantaurum, v. 78, n. 2, p. 309-314, 1990.

[24] NGUYEN, T. H.; FLETE, G. H.; ROGERS, P. L. Composition of the cell walls of several yeast species. Applied Microbiology and Biotechnology, v. 50, n. 3, p. 206212, 1998.
[25] OHNO, N. ; UCHIYAMA, M.; TSUZUKI, A.; TOKUNAKA, K.; MIURA, N. N. ; ADACHI, Y.; AIZAWA, M.W.; TAMURA, H.; TANAKA, S.; YADOMAE, T. Solubilization of yeast cell-wall beto-(1,3)-D-glucan by sodium hypochlorite oxidation and dimethyl sulfoxide extraction. Carbohydrate Research, v. 316, n. 1-4, p. 161-172, 1999.

[26] OUARNIER, N. A.; QUEMENER, B.; BERTRAND, D.; BOIVIN, P. Application of high performance anion exchange chromatography to the study of carbohydrate changes in barley during malting. Journal of the Institute of Brewing, v. 106, n. 1, p. 45-52, 2000.

[27] OZDEMIR, M.; GENÇ, H. Bet $\alpha$-glucan contents of cereal grains grown in Turkey. Energy Education Science and Technology, v. 7, n. 1, p. 10-17, 2001.

[28] PETERSON, D. M.; WESENBERG, D. M.; BURRUP, D. E. $\beta$-Glucan content and its relationship to agronomic characteristics in elite oat germplasm. Crop Science, v. 35, n. 4, p. 965-970, 1995.

[29] PROSKY, L.; ASP, N. G.; SCHWEIZER, T. F.; DEVRIES, J. W.; FURDA, I. Determination of insoluble, soluble, and total dietary fiber in foods and food-products Interlaboratory study. Journal of Association of Official Analytical Chemistry, v. 71, n. 5, p. 1017-1023, 1988.

[30] SAASTAMOINEN, M.; PLAAMI, S.; KUMPULAINEN, J. Bet $\alpha-$ glucan and phytic acid content of oats cultivated in Finland. Acta Agriculturae Scandinavica, v. 42, n. 1, p. 6-11, 1992.

[31] SANDULA, J.; KOGAN, G.; KACURAKOVA, M.; MACHOVA, E. Microbial (1-3)-bet $\alpha-D$-glucans, their preparation, physico-chemical characterization and immunomodulatory activity. Carbohydrate Polymers, v. 38, n. 3, p. 247-253, 1999.

[32] SCHOFFELMEER, E. A. M.; KLIS, F. M.; SIETSMA, J. H.; CORNELISSEN, B. J. C. The cell wall of Fusarium oxysporum. Fungal Genetics and Biology, v. 27, n. 2/ 3, p. 275-282, 1999.

[33] VIS, R. B.; LORENZ, K. Bet $\alpha$-glucans: importance in brewing and methods of analysis. Lebensmittel-Wissen undTechnologie, v. 30, n. 4, p. 331-336, 1997.

[34] WASSER, S. P. ; WEIS, A. L. Therapeutic effects of substances occurring in higher basidiomycetes mushrooms: A modern perspective. Critical Review of Immunology, v. 19, n. 1, p. 65-96, 1999.

[35] WELCH, R.W.; BROWN, J.C.W.; LEGGETT, J.M. Interspecific and intraspecific variation in grain and great characteristics of wild oat (Avena) species: very high great $(1,3),(1,4)$-bet $\alpha$-glucan in an Avena atlantica genotype. Journal of Cereal Science, v. 31, n. 3, p. 273-279, 2000. 